\title{
Differential characterization of myogenic satellite cells with linolenic and retinoic acid in the presence of thiazolidinediones from prepubertal Korean black goats
}

\author{
S. Subi ${ }^{1, a}$, S. J. Lee, ${ }^{1, a}$, S. Shiwani ${ }^{1}$, and N. K. Singh ${ }^{2, *}$
}

* Corresponding Author: N. K. Singh Tel: +91-9554786939, E-mail: naresh2101@gmail.com

${ }^{1}$ College of Animal life sciences, Kangwon National University, Chuncheon 24341, Korea

2 Department of Veterinary Surgery and Radiology, Faculty of Veterinary and Animal Sciences, Institute of Agricultural Sciences, Banaras Hindu University, Varanasi-221005, Uttar Pradesh, India

a These two authorscontribute equally to this work.

ORCID

S. Subi

https://orcid.org/0000-0003-1174-9810

S. J. Lee

https://orcid.org/0000-0001-9348-8356

S. Shiwani

https://orcid.org/0000-0002-6852-8384

N. K. Singh

https://orcid.org/0000-0001-6360-1610

Submitted Apr 2, 2017; Revised Jun 15, 2017; Accepted Aug 31, 2017
Objective: Myogenic satellite cells were isolated from semitendinosus muscle of prepubertal Korean black goat to observe the differential effect of linolenic and retinoic acid in thepresence of thiazolidinediones (TZD) and also to observe the production insulin sensitive preadipocyte.

Methods: Cells were characterized for their stemness with cluster of differentiation 34 (CD34), CD13, CD106, CD44, Vimentin surface markers using flow cytometry. Cells characterized themselves as possessing significant $(\mathrm{p}<0.05)$ levels of CD13, CD34, CD106, Vimentin revealing their stemness potential. Goat myogenic satellite cells also exhibited CD44, indicating that they possessed a $\%$ of stemness factors of adipose lineage apart from their inherent stemness of paxillin factors $3 / 7$.

Results: Cells during proliferation stayed absolutely and firmly within the myogenic fate without any external cues and continued to show a significant $(\mathrm{p}<0.05)$ fusion index \% to express myogenic differentiation, myosin heavy chain, and smooth muscle actin in $2 \%$ horse serum. However, confluent myogenic satellite cells were the ones easily turning into adipogenic lineage. Intriguingly, upregulation in adipose specific genetic markers such as peroxisome proliferation-activated receptor $\gamma$, adiponectin, lipoprotein lipase, and CCAAT/ enhancer binding protein a were observed and confirmed in all given treatments. However, the amount of adipogenesis was found to be statistically significant $(\mathrm{p}<0.01)$ with linolenic acid as compared to retinoic acid in combination with TZD's.

Conclusion: Retinoic acid was found to produce smaller preadipocytes which have been assumed to have insulin sensitization and hence retinoic acid could be used as a potential agent to sensitize tissues to insulin in combination with TZD's to treat diabetic conditions in humans and animals in future.

Keywords: Goat Muscle Satellite Cells; Thiazolidinedione; Linolenic Acid; Retinoic Acids; Adipogenesis

\section{INTRODUCTION}

The multinucleated myotubes, which are generated by the fusion of several mononucleated myoblasts during embryonic and fetal developments, are the functional unit of skeletal muscle that generates force by their contraction [1]. The regeneration of myotubes after damage or injury is considered to be very rapid and gets established within weeks with the help of precursor cells, such as satellite cells or muscle derived stem cells (MDSCs). The skeletal muscle satellite cells are non differentiated quiescent cells, which are known to arise from embryonic mesoderm and locate themselves on the surface of myofibers beneath the basal membrane [1]. During injury, quiescent satellite cells get activated to proliferate and produce nascent myofibers for the efficient repair [2]. However, depending on the environmental 
conditions, satellite cells are also capable of differentiating into certain other cell type like adipocytes, osteocytes, chondrocytes etc. [3]. Furthermore, marbling in muscle enhances flavor and nutritional value of meats and those appeals to the consumers. Therefore, in the past several researchers have tried to increase marbling with the use of various PPAR-gamma agonists. Thiazolidinediones (TZD's) are one such adipogenic inducer that has been so far used as antidiabetic agents in humans [4]. Since retinoic acid (RA) has been found to be liposoluble vitamin A (retinol) and its acidic metabolite acts as a ligand for transcription factors of the retinoic acid receptors (RAR; RAR $\alpha$, RAR $\beta$, and RAR $\gamma$ ) [5]. Furthermore, it was also shown that RA could serve as a ligand for the nuclear receptor peroxisome proliferation-activated receptor $\beta / \delta$ $(\operatorname{PPAR} \beta / \delta)[6]$ and probably favors binding to $\operatorname{PPAR} \beta / \gamma$ to induce anti-apoptotic response in higher concentrations of fatty acid binding protein-5 (FABP5) [6]. We therefore assume and consider TZD's as one the strong agents to initiate and produce FABP5 concentration. However, RA in combination with the TZD's could attain increased levels of adipogenesis as compared to the use of TZD's alone. Furthermore, this combination could bind PPAR $\beta / \gamma$ comfortably to initiate a better adipogenic phenomenon. We therefore, designed the study to investigate the similar phenomenon to happen on Korean black goat myogenic satellite cells (GMSCs) involving the lipid and glucose metabolism when the RA and linolenic acid combined with thiozolidienediones.

Due to the paucity of literature on this subject instigated us to investigate the possible adipogenic differentiation of GMSCs and potential insulin sensitization (through the production of smaller lipid droplets) by RA with TZD’s.

\section{MATERIALS AND METHODS}

\section{Chemicals, antibodies and primers}

We purchased Dulbecco's modified Eagle's medium (DMEM), penicillin and streptomycin (PS) from Welgene (Dalseogu, Daegu, Korea), fetal bovine serum (FBS) from HyClone Laboratories, Inc (Logan, UT, USA), horse serum (HS) from Gibco Technologies Inc (Grand Island, NY, USA), Power cDNA synthesis kit from Intron biotechnology and primers from Macrogen. The RA, linolenic acid, pigskin gelatin, dexamethasone, insulin, biotin, ascorbic acid, pantothenic acid, ascorbic acid and protease (Pronase [P5147]) were obtained from Sigma Aldrich (Seoul, Korea). Xylazine and Ketamine were obtained from Yuhan Co., Seoul, Korea. GMSC's were isolated from 90 days old goats.

\section{Animal rearing and tissue collection}

Eight Korean black goats (approximately 90 days old) were utilized for the experiment and maintained in the animal house meant especially for animal experimentation (having all the relevant facilities required ethically for animal experimentation) of the college of animal life sciences, Kangwon National University, Republic of Korea. All these procedures were carried out as per the ethical guidelines and regulations for animal experimentation of Kangwon National University, Republic of Korea. A total of four goats were fed with high fat rich diet to gain fat generalized body pad relatively faster. The ingredient composition of the concentrate fed to treatment group is given in Table 1 together with analyzed chemical composition of the concentrate and hay. The Korean black goats in the control group had access to Timothy hay on an ad libitum basis. Goats were dewormed initially with broad-spectrum anthelmintics and were vaccinated against prevalent contagious diseases. The body weight (BW) of individual goats was recorded at the start and thereafter at fortnightly intervals until 90 days of the trial in the morning before feeding, in order to assess changes in BW and average daily gain. The biopsied muscle tissues were first kept in cold phosphate buffered saline (PBS) containing 5\% antibiotics, until the isolation of GMSC's. Finally, the surgical opening was closed as per the routine surgical maneuver. Postoperative care and management was done under the supervision of a veterinary surgeon for a period of one week.

\section{Goat myogenic satellite cells isolation}

GMSC's were isolated from semitendinosus muscle as described the literature [7] with some modifications. Isolated primary myoblasts were stored in liquid nitrogen until used.

\section{Flow cytometry}

We performed flow cytometric analysis as demonstrated by the literature [8]. Briefly, to assess expressions of specific markers, we harvested cells using citrate saline buffer $(0.134 \mathrm{~mol} / \mathrm{L}$ $\mathrm{KCl}, 0.015 \mathrm{~mol} / \mathrm{L} \mathrm{Na}$ citrate), and washed them twice with cold PBS containing $0.002 \%$ ethylenediaminetetraacetic acid (EDTA) and $10 \mathrm{mmol} / \mathrm{L} \mathrm{NaN}_{3}$. The cells fixed with $4 \%$ paraformaldehyde were incubated with primary antibodies, which were diluted in 1:50 ratio in PBS containing 0.5\% bovine serum albumin (BSA), for $1 \mathrm{~h}$ on ice, followed by a wash with

Table 1. Ingredients and analyzed chemical composition of concentrate and Timothy hay

\begin{tabular}{llcc}
\hline SI. No. & Composition & Concentrate & Timothy hay \\
\hline 1. & Dry matter & 881 & 931 \\
2. & Crude protein & 166 & 93 \\
3. & Ether extract & 45 & 20 \\
4. & NDF & 343 & 664 \\
5. & ADF & 123 & 418 \\
6. & Ash & 95 & 107 \\
7. & Ca & 12 & 2 \\
8. & P & 5 & 2 \\
\hline
\end{tabular}

NDF, neutral detergent fiber; $A D F$, acid detergent fiber. 
PBS-BSA. The non-labelled primary antibodies were visualized by using 1:50 diluted mouse anti-goat immunoglobulins $G$ (secondary antibodies) conjugated to fluorescein-isothiocyanate. After washing, we analyzed cell suspensions using a flow cytometer (Epics XL analyzer; Coulter Corporation, Miami, FL, USA) after the addition of $5 \mathrm{~mL}$ of a $1-\mathrm{mg} / \mathrm{mL}$ solution of propidium iodide to exclude nonviable cells. We analyzed at least $1 \times 10^{4}$ cells/sample.

\section{Doubling time}

The isolated primary cells were counted with a hemocytometer by diluting with trypan blue. The same isolated primary cells $\left(1 \times 10^{5}\right)$ were seeded in a $100 \mathrm{~mm}$ dish containing DMEM supplemented with 20\% FBS and 1.1\% PS for proliferation. The media was changed in 2 to 3 days interval until $80 \%$ confluency. At this point, the cells were passaged with the help of $0.25 \%$ trypsin and counted to calculate the final number of cells. A certain portion of these cells were again reseeded and carried on till passage 4 , subsequently the culturing time was also recorded. The doubling time was calculated according to the literature [9] with certain modifications. i.e.,

$$
\begin{aligned}
& \mathrm{CD}=\ln \left(\mathrm{N}_{\mathrm{f}} / \mathrm{N}_{\mathrm{i}}\right) / \ln (2) \\
& \mathrm{DT}=\mathrm{CT} / \mathrm{CD}
\end{aligned}
$$

Where, DT is the cell-doubling time; CT, the cell culture time; $\mathrm{CD}$, the cell-doubling number; $\mathrm{N}_{\mathrm{f}}$, the final number of cells; $\mathrm{N}_{\mathrm{i}}$, the initial number of cells.

\section{Cell culture}

The isolated GMSC's were characterized, by flow cytometric analysis, and proliferated in DMEM supplemented with $10 \%$ FBS and $1.1 \%$ PS and incubated at $37^{\circ} \mathrm{C}$ in $5 \% \mathrm{CO}_{2}$ incubator. After reaching $70 \%$ to $80 \%$ confluency, the cells were subcultured with the help of trypsin EDTA. The 2nd passage cells were used for differentiation experiments.

For myogenic differentiation of GMSC's, $1 \times 10^{6}$ cells were seeded in $60 \mathrm{~mm}$ dish and cultured with DMEM containing $2 \% \mathrm{HS}$ and $1.1 \%$ PS. Media were being changed in every 48 hours and samples were taken on days 0,4 , and 8 .

For adipogenic differentiation of GMSC's, $1 \times 10^{6}$ cells were seeded into $60 \mathrm{~mm}$ culture dish. After getting $80 \%$ confluency, the cells were induced by adipogenic differentiation media comprising, DMEM containing $2 \% \mathrm{HS}, 1.1 \%$ PS, $50 \mu \mathrm{M}$ ascorbic acid, $33 \mu \mathrm{M}$ biotin, $10 \mu \mathrm{M}$ acetic acid, $17 \mu \mathrm{M}$ pantothenic acid, $0.5 \mu \mathrm{M}$ 3-Isobutyl-1-methylxanthine, $1 \mu \mathrm{M}$ dexamethasone, $10 \mu \mathrm{M}$ insulin, $10 \mu \mathrm{M}$ 2, 4-TZD. In addition, 2 dishes were treated with $100 \mathrm{mM} \mathrm{LA}$ and 2 with $1 \mathrm{nM} \mathrm{RA}$. Media were changed in every 48 hours with respective concentrations of TZD, LA, and RA in appropriate dishes till the final day of induction. Samples were harvested for RNA isolation on days 0,4 , and 8 . Staining samples were washed with PBS and fixed with $4 \%$ paraformaldehyde for oil red O (ORO) staining.

\section{Fusion index}

The myogenic differentiated cells were collected on 0,4 , and 8 days of culture. The collected samples were stained with hematoxyline (Sigma Aldrich, Korea) nuclei staining dye and scored the total number of nuclei as well as the number of nuclei incorporated with the myotubes within 10 different microscopic fields (400× magnification). The myoblasts containing at least 3 nuclei are counted as myotubes, further the fusion index for each sample was calculated as the percentage of nuclei incorporated to the myotubes compared to the total number of nuclei scored over the particular microscopic field [4].

\section{Adipocyte stain uptake and oil red $O$ staining}

Measurement of stain retained by the adipoblasts was done to determine the increase in the number or size of the adipoblasts. The ORO staining was performed to confirm the adipogenic differentiation of GMSC's. The working stain solution was prepared by diluting 6 parts of stock stain solution ( $0.5 \%$ ORO in isopropanol) in 4 parts of deionized wzater. We followed the protocol of Singh et al [4] with some modifications. Briefly, the formalin fixed cell samples were treated with stain for 10 min. The excess stain was removed, washed with DW and dried before capturing the stained cells under a light microscope. Finally the stain was eluted using $100 \%$ isopropanol and its optical density was measured at $500 \mathrm{~nm}$ by setting $100 \%$ isopropanol as blank [10].

\section{Reverse-transcriptase polymerase chain reaction}

Total RNA was isolated from each sample using TRIZOL reagent according to the standard protocols. Total RNA $(8 \mu \mathrm{g})$ was taken to synthesis cDNA using the power cDNA synthesis kit according to the manufacturer's instructions. So synthesized cDNA was used as template in polymerase chain reaction (PCR), which was performed with Takara PCR kit on the specific primer conditions (initial denaturation at $95^{\circ} \mathrm{C}$ for 5 minutes, followed by 35 cycles of $95^{\circ} \mathrm{C}$ for $40 \mathrm{~s}$, amplification temperature (Temp, Table 2) for $1 \mathrm{~min}, 72^{\circ} \mathrm{C}$ for $40 \mathrm{~s}$ and a final elongation at $72^{\circ} \mathrm{C}$ for $8 \mathrm{~min}$ ). Amplified templates were resolved in $1 \%$ to $1.2 \%$ agarose gel stained with Ethidium bromide and visualized under UV gel documentation system.

\section{Statistical analysis}

The treatments at different time intervals were analyzed by analysis of variance, using the statistical software package SAS (SAS Inst. Inc., Cary, NC, USA). The significant differences were determined $(\mathrm{p}<0.05, \mathrm{p}<0.01)$ by the Duncan's multiple range tests using SAS. 
Table 2. Primer details used in reverse-transcriptase polymerase chain reaction

\begin{tabular}{|c|c|c|c|c|}
\hline Gene & Accession No. & Sequences & & Temp $\left({ }^{\circ} \mathrm{C}\right)$ \\
\hline Adipo Q & BC140488 & 5' GATCCAGGTCTTGTTGGTCCTAA 3' & 5' GAGCGGTATACATAGGCACTTTCTC 3' & 60 \\
\hline$L P L$ & M16966 & 5' TACCCTGCCTGAAGTTTCCAC 3' & 5' CCCAGTTTCAGCCAGACTTTC 3' & 60 \\
\hline$C / E B P \alpha$ & BC149006. 1 & 5' AGTCCGTGGACAAGAACAGC 3' & 5' GGTCATTGTCACTGGTCAGC 3' & 53 \\
\hline$P P A R \gamma$ & NM001100921.1 & $5^{\prime}$ ACGGGAAAGACGACAGACAAA 3' & $5^{\prime}$ GACGGAGCGAAACTGACACC $3^{\prime}$ & 59 \\
\hline$M H C$ & NM 017240 & $5^{\prime}$ AGAAGGAGCAGGACACCAGC $3^{\prime}$ & 5' GGTCTCAGGGCTTCACAGGC 3' & 57 \\
\hline MyoD & NM 010866. 2 & $5^{\prime}$ GACAGGGAGGAGGGGTAGAG 3' & 5' TGCTGTCTCAAAGGAGCAGA 3' & 59 \\
\hline aSMA & BC158550.1 & 5' GATCACCATCGGGAATGAACGC 3' & 5' CTTAGAAGCATTTGCGGTGGAC 3' & 60 \\
\hline
\end{tabular}

Adipo $Q$, adiponectin; $L P L$, lipoprotein lipase; C/EBPa, CCAAT/enhancer binding protein $\alpha$; PPAR $\gamma$, peroxisome proliferation-activated receptor $\gamma ; M H C$, myosin heavy chain; $M y O D$, myogenic differentiation; $\alpha S M A$, $\alpha$-smooth muscle actin.

\section{RESULTS}

\section{Cytofluorimetric analysis of purified GMSC's and it} proliferative property

After purification by differential trypsinization and adherence, we further cultured cells until passage 2 . We harvested passage 2 cells and analyzed them by flow cytometry to evaluate stemness-related CD markers, and to verify whether these cells were able to maintain the characteristics of stemness. As shown in Figure 1A, at passage 2, purified GMSC's expressed significant levels of CD106 and CD44 ( $<<0.01)$ (stemness factors) and $\mathrm{CD} 13$ and $\mathrm{CD} 34(\mathrm{p}<0.05)$ (specific markers for satellite cells). $\alpha$-Smooth muscle actin ( $\alpha$-SMA) and vimentin was also observed to significant relatively. The results shown represent three independent experiments.
Cellular morphology of purified GMSC's and theirdoubling time during proliferation

Purified GMSC's appeared as tiny and small round spheres initially when seeded, but later it adhered strongly to the dish surface. However, more than 95\% adherence was apparent only after 24 hours. From day 2 onwards the cells stretched to give fusiform appearance. Cellular extension called filapodia's helped each cell to elongate and adhere to the culture plates and probably provided enough strength to proliferate and divide. The process of proliferation continued, and cells assumed spindle shape with elongated nucleus. All the cells had a distinct nucleus, sometimes with nucleolus and abundant cytoplasm. The viability of cells at every stage of proliferation was $>95 \%$. We dislodged the cellular colonies once we realized that the cells have reached a stage of contact inhibition and further distributed equally to expand them further. After the
A
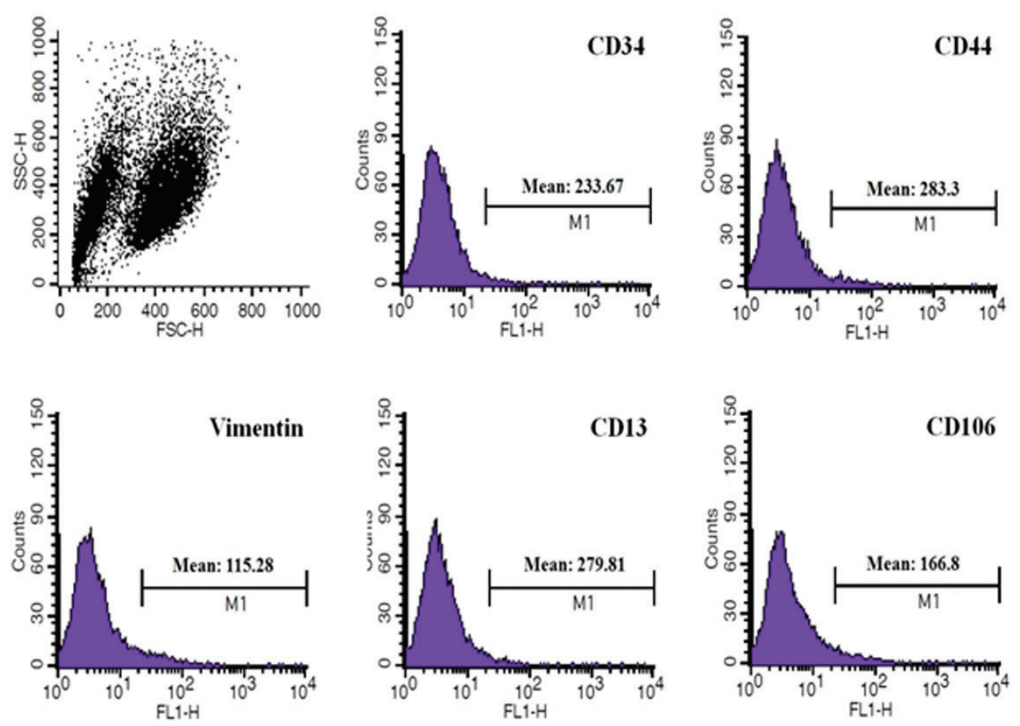
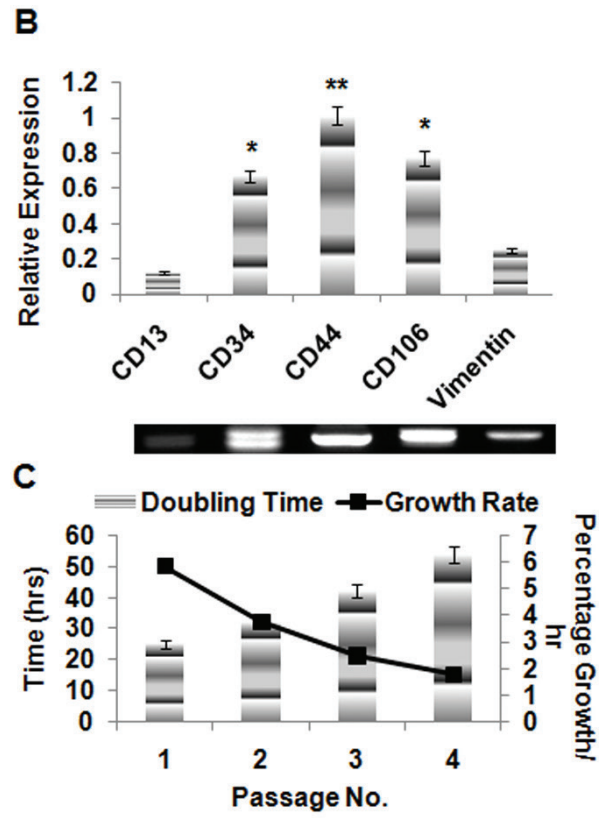

Figure 1. Characterization of isolated myosatellite cells. Characterization of primary capra myosatellite cells using the stem cell as well as myoblast specific markers such as cluster of differentiation 13 (CD 13), CD34, CD44, CD106 and vimentin by flow cytometry (A) and reverse-transcriptase polymerase chain reaction (B). Significant expressions of CD34, CD44, and CD 106 were detected in the myoblast cells ( $\left.{ }^{*} p<0.05,{ }^{* *} p<0.01\right)$. (C) The graph shows the changes in doubling time and percentage proliferation rate of isolated capra myoblasts at each passage. 
first passage the cells assumed a faster growth relatively and assumed fusiform-like morphology and showed $90 \%$ to $95 \%$ confluent state within 2 to $3 \mathrm{~d}$ (Figure 2A). The isolated myoblast cells were initially round in shape in suspending media, however it became spindle as soon as it got attached to the bottom of the dish. The attached cells grew rapidly and were passaged upon attaining $>95 \%$ confluent stage. The doubling time was calculated while subculturing and it showed increased mitotic time with each passage. Finally, for differential experiments we took second passage cells.

\section{Determination of myogenic lineage differentiation of purified GMSC's}

GMSC's formed complete multi-nucleated myofiber morphology when cultured under limited serum conditions (2\% HS). The myotube formation initiated since the fourth day (Figure 2A) of culturing with limited serum, and on further days, the length of the myotubes increased gradually. From the fusion index data (Figure 2C), it is clear that the nuclei count in the myotube was increasing significantly $(\mathrm{p}<0.05)$ according to the time, since day 4 . On reverse-transcriptase polymerase chain reaction (RT-PCR), the myogenic marker genes such as myogenic differentiation (MyoD), aSMA, and myosin heavy chain (MHC) showed different expressions on different time periods. The MyoD expression was not detected much within these periods, however, a slight increase in its expression was noted on 4th day. The contractile gene, aSMA had a higher expression in the initial days, however, it declined rapidly on the 4th day and increased a bit on the 8th day samples. Further, the expression of MHC was detected lesser on the 4th day, however its maximum expression $(\mathrm{p}<0.01)$ was detected on the 8th day of the experiment (Figure 2B).

\section{Determination of thiazolidenediones (PPAR-gamma ligand) adipogenic potentialon purified GMSC's alone and in combination with either retinoic acid orlinolenic acid}

The preconfluent cells, when treated withwith TZD, LA+TZD, and RA+TZD did not formnot form myotubes. Rather, they intended to acquire ring shaped morphology with increased synthesis and accumulation of triglycerides (Figure 3A). The triglyceride accumulation within the cells was detected by ORO staining (Figure $3 \mathrm{~A}$ ) and elution index was found to be significant $(\mathrm{p}<0.01)$ in LA+TZD compared to TZD and RA+ TZD treated cells (Figure 3B). Data obtained further supported the results obtained by RT-PCR (Figure 4). Adipose specific genes such as PPAR $\gamma$, adiponectin (Adipo Q), and lipoprotein lipase (LPL) have shown progressive expressions upon incubation, however, highest expressions $(\mathrm{p}<0.01)$ in $\mathrm{LA}+\mathrm{TZD}$ treated cells than TZD and RA+TZD were noted. Similarly, the terminal adipogenic differentiation marker CCAAT/enhancer binding protein $\alpha(\mathrm{C} / \mathrm{EBP} \alpha)$ was found to be highly
$\mathbf{A}$

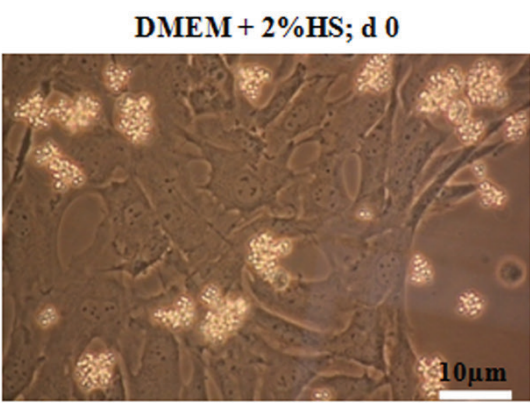

DMEM + $2 \% H S ; d ~ 4$

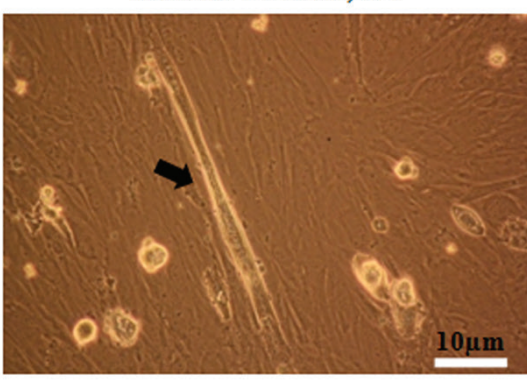

DMEM $+2 \% H S ;$ d 2

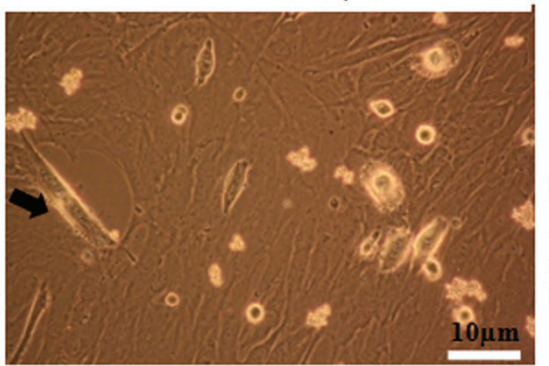

DMEM $+2 \%$ HS; $\mathrm{d} S$

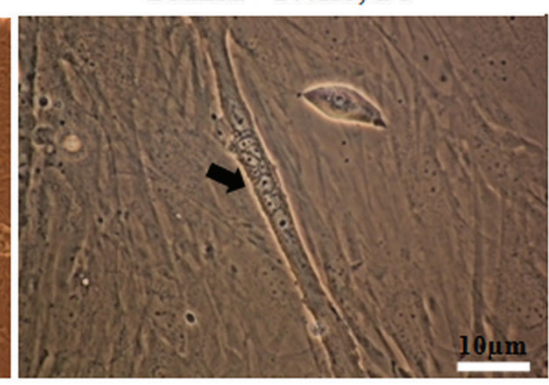

B

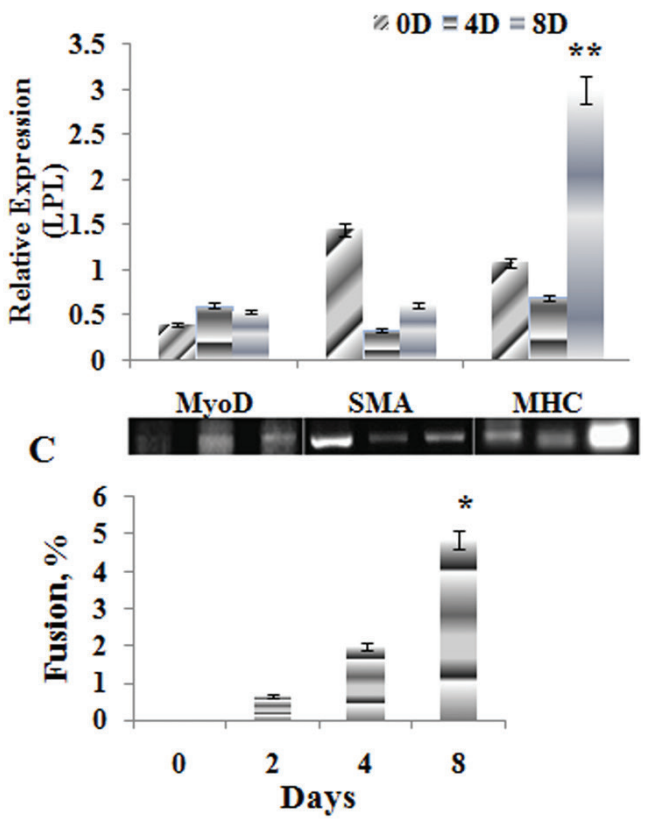

Figure 2. Morphological changes and fusion index during myogenic differentiation. (A) the morphological changes observed under light microscope in the myogenic control myosatellite cells cultured under limited serum conditions during $0,2,4$, and 8 days, where, arrows show the myotube formation. (B) Relative expressions of myogenic genes detected through reverse-transcriptase polymerase chain reaction. The bands attained were analyzed using ImageJ, and normalized to the internal loading control GAPDH. ( ${ }^{* *} \mathrm{p}<0.01$ ). (C) The chart shows the fusion index (nuclei count in a single myotube) during the $0,2,4$, and 8th days of myogenic differentiation. GAPDH, glyceraldehyde-3-phosphate-dehydrogenase. 
I

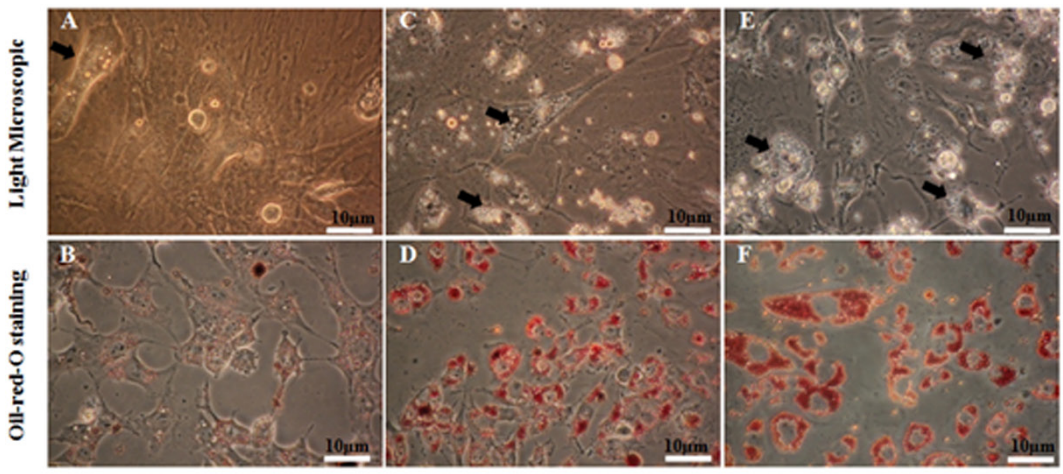

II

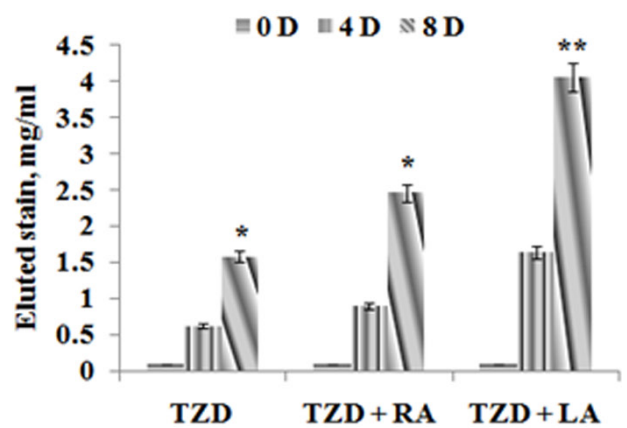

Figure 3. Microphotographic view, ORO staining and elution index of differentiating myoblasts. I: Microscopic $(A, C, E)$ and ORO (B, D, F) stained images of myosatellite cells under adipogenic inductions (Day 8). Morphological changes and stained lipid droplets were visible in TZD ( $A, B), R A+T Z D(C, D)$ and LA+TZD (E, F) induced myoblast cells, under inverted light microscope. II: The differentiating myoblasts were stained with ORO for evaluating the lipid droplet accumulation. Elution indexes of each treatment were quantified by calculating optical density of the isopropanol eluted stain. Whereas, most significant staining was detected under TZD+LA treatment. ( ${ }^{*}$ $\left.p<0.05,{ }^{* *} p<0.01\right)$. ORO staining, oil red 0 staining; TZD, thiazolidinediones.

$\mathbf{A}$

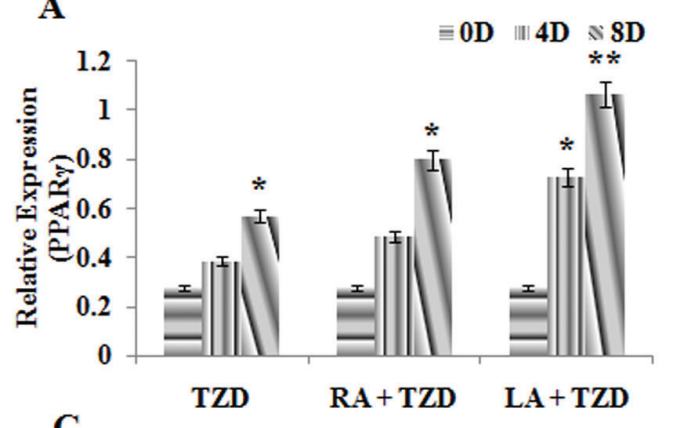

C

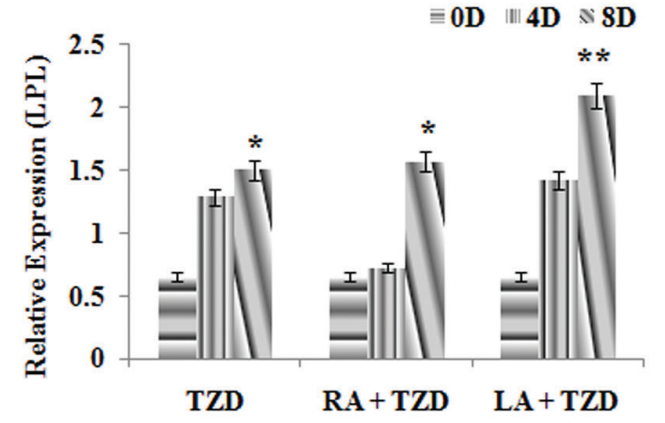

B
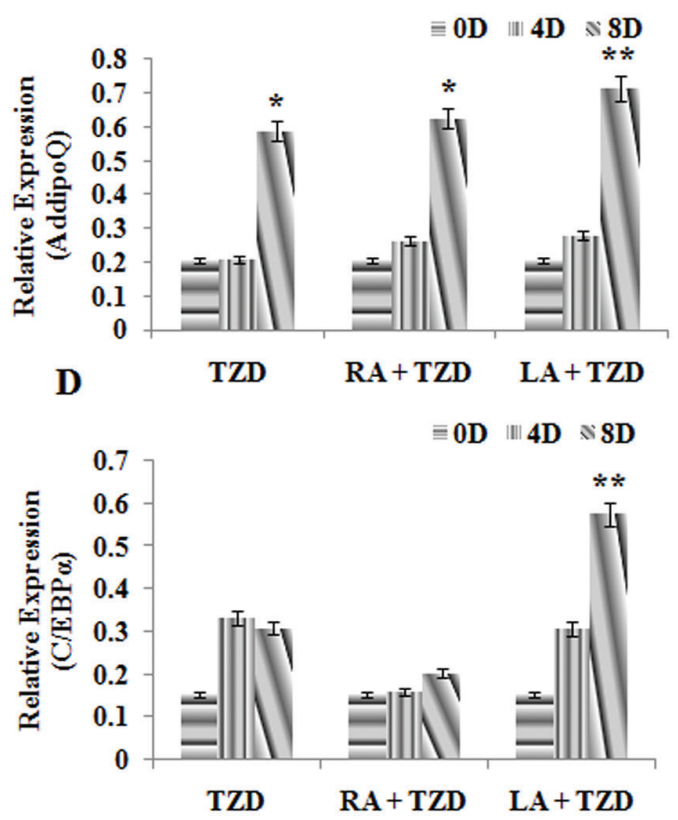

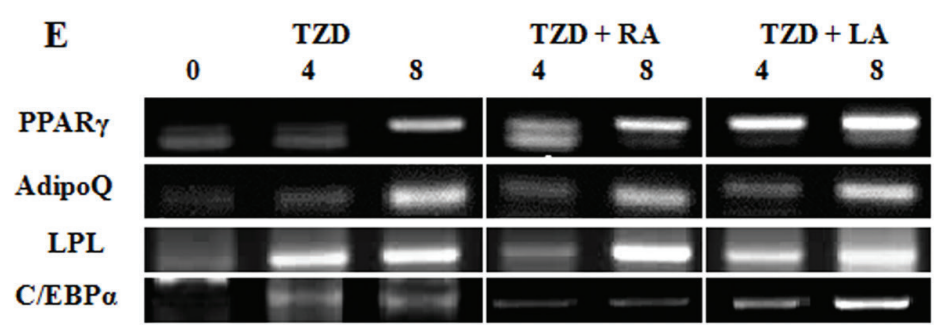

Figure 4. Genetic expression analyses of adipose induced myoblast cells. The graphs show the relative expressions of PPARy (A), Adipo Q (B), LPL (C) and C/EBPa (D) in myoblasts, respectively in 0,4 , and 8 days under TZD, RA+TZD and LA+TZD treatments. Highly significant expressions of adipocyte genes were observed under TZD+LA treatment compared to the other two. Moreover, predominant expression of terminal adipocyte differentiation marker (C/EBPa) was monitored under this treatment. Bands of each reverse-transcriptase polymerase chain reaction product were analyzed and normalized to internal loading control GAPDH using ImageJ software. $\left({ }^{*}: p<0.05,{ }^{* *}\right.$ : p < 0.01). PPAR $\gamma$, peroxisome proliferation-activated receptor $\gamma$; Adipo Q, adiponectin; LPL, lipoprotein lipase; C/EBPa, CCAAT/enhancer binding protein $\alpha$; TZD, thiazolidinediones, GAPDH, glyceraldehyde-3-phosphate-dehydrogenase. 
expressed $(\mathrm{p}<0.01)$ in the LA+TZD as compared to TZD alone and RA+TZD treatments.

\section{DISCUSSION}

CD34, a cluster of differentiation gets activated through the alternative splicing just before the end of transcription and has been demonstrated to regulate differentiation and lineage primed progenitor compartments in skeletal muscle cells and has been associated with vascular stem cells [11] as well. GMSC's had significant $(\mathrm{p}<0.01)$ expression levels of CD34, we believe that these GMSC's possess intense immune related components as $\mathrm{CD} 34$ has been shown to help T cells to enter lymph nodes [12]. It further provides us an inkling that the many issues that haunt us and remain unaddressed about the role of mammary epithelium in regulating immune responses (in handling T-cell related queries) during trauma and injury would probably be unfurled provided further investigation are done in this direction. Moreover, due to fact that muscles posses plentiful of common factors with mammary epithelial and myoepithelial stem cells and therefore we believe that these cells could also pave a way to search regenerative possibilities of mammary epithelium and myoepithelium with muscle cells [13].

CD13 is one of the pluripotency associated markers whose expression in the stem cells helps to retain the differentiation capacity even after indefinite proliferation. Besides its expression has also been reported in mouse multipotent adult progenitor cells and MDSCs [14]. However, its major function is to regulate the cell motility and chemotaxis. In addition, CD 106 or vascular adhesion molecule-1 (VCAM-1) are also potential markers of quiescent cells [15]. These adhesion molecules may function in the adhesion of the satellite cells to the basal lamina of the myofibre and participate in the migration of satellite cells in response to different stimuli. The VCAM is expressed in both myofibres and satellite cells, where as it is limited to satellite cells in adult muscle and it mediates interaction of satellite cells to leukocytes following injury [15]. Further, GMSC's found to be expressing vimentin gene relatively to a lesser extent, as vimentin is an intermediate filament expressed in mesenchymal cells, which governs the structural maintenance of the cells [16]. Moreover, vimentin expression was reported in myoblasts and has been associated with regeneration and during muscular repair and that shows that its on and off expressions are event related and has strongly something to do with the regeneration and repair phenomenon [17].

The cells of control group, however, did not deviate to any other lineage than myogenic lineage when cultured solely in DMEM containing 2\% HS revealed that GMSC's would only alter its lineage when strong factors would come forward to upregulate alternate lineage gene markers. The expressions of myogenic specific genes like MyoD, aSMA, and MHC were detected during proliferation and differentiation. $\mathrm{MyoD}$ is a member of myogenic regulatory factor, which is one of the initially expressed markers in myogenesis and also has a role to play in the activation of satellite cells [18] in the event of injury and thereby initiates the process of repair and regeneration. Moreover, it induces the initial differentiation of myoblasts as it has been shown and demonstrated earlier that it is quite capable in relocating chromatin in the lineage restricted loci [19]. We also witnessed and observed that MyoD expression were strong during the initial stages of differentiation. However, we observed its declining expressions in the formed myotubes.

Similarly the aSMA, which is a vascular isoform of smooth muscle actin, plays an important role in the satellite cell differentiation, were also evident throughout the period of proliferation and differentiation showed the integrity of GMSC's, as aSMA represents that their mere presence in some cellular content relates it to the existence of contractile protein necessary for contraction [20]. And its associated with the GMSC's apparently reveals that these cells descendents of myogenic lineage and of course are adult and differentiated cells. Moreover, aSMA was noticed dominantly only while the myoblast differentiation and a little expression was detected after the formation contractile myotubes [21] in the primary mouse myoblasts. On the other hand, we observed significantly $(\mathrm{p}<0.01)$ upregulated MHC expressions in the GMSC's during differentiation in GMSC's. MHC is a thick filament component and a predominant protein in skeletal muscle, which is related to contraction [22] shows that the MHC components starts developing and concentrating and gets clearly and strongly expressed only while myoblast starts turning into myotubes as well as in the fully differentiated myotubes [23].

The RA is derived from the liposoluble vitamin A (retinol). Molecular basis of vitamin A action was elucidated almost three decades back and was demonstrated that its acidic metabolite, RA, acts as a ligand for transcription factors of the RAR nuclear receptor superfamily and claimed RA as a potent transcriptional activator than repressor. All trans-retinoic acid have been demonstrated to regulate gene expression and all vitamin A metabolite (RA) regulates gene expression by activating specific members of the superfamily of transcription factors known as nuclear hormone receptors. It has long been established that many biological activities of RA are mediated by RAR (RARa, RAR $\beta$, and RAR $\gamma$ ) [5]. However, recently it was shown that in addition to activating RARs, RA can also serve as a ligand for the nuclear receptor PPAR $\beta / \delta[6]$. However, it has been also observed that retinoic acid favours binding to $\operatorname{PPAR} \beta / \gamma$, which in turn can induce anti-apoptotic and proliferative responses only when FABP5 concentration becomes higher [6]. We therefore, assume and consider TZD's as one the strong agents to initiate and produce FABP5 concentration and because we observed that when we used RA in combination with the TZD's, level of adipogenesis increased 
significantly as compared to the use of TZD's alone. RA in combination with TZD's has probably become the right choice of combination for binding with PPAR $\beta / \gamma$ comfortably to initiate a better adipogenic phenomenon. We, therefore, report that as the repertoire of direct targets for PPAR $\beta / \delta$ includes genes involved in lipid and glucose metabolism, and that activation of this receptor increases lipid catabolism in skeletal muscle and adipose tissue and therefore prevents the development of obesity and improves insulin sensitivity in obesity [24]. Consequently, we propose PPAR $\beta / \delta$ agonists may be efficacious agents in the treatment of the metabolic syndrome and it has also been observed earlier that RA functions as a PPAR $\beta / \delta$ ligand, which raises the intriguing possibility of its salient roles in the regulation of sugar and lipid metabolism and disposition [25].

As far as TZD's pharmacological actions are concerned, TZD's alter PPAR $\gamma$ expression to reduce insulin resistance through adipogenic differentiation of myoblasts. It has been demonstrated that in the absence of increased energy storage, TZD's would be expected to produce more fat cells of a smaller average size, which are usually more sensitive to insulin. Such a response in GMSC's would be expected to produce greater insulin-dependent glucose uptake [26]. Moreover, as insulin is a powerful antilipolytic agent, smaller fat cells with increased insulin sensitivity would be expected to have relatively lower rates of lipolysis, because high levels of free fatty acids may be causally involved in the induction of insulin resistance, this could affect insulin sensitivity at distant sites such as muscle and liver [27]. This mechanism would also be compatible with the so-called randle effect, where the tendency of muscle to utilize glucose as an energy source is inversely correlated with the use of fatty acids as an energy source. There are also reports that TZD administration to rodents greatly increases the amounts of brown adipose tissue [28]. Considering that this tissue functions to dissipate energy through the oxidation of fatty acids, it could also reduce circulating lipid levels and have a beneficial effect on insulin sensitivity.

Moreover, the TZD's and its subfamily, though are PPARgamma stimulators but normally does not work as other ligands that have more full agonist activity on PPAR $\gamma /$ retinoic $\mathrm{X}$ receptor (RXR) heterodimers. Therefore, TZD's usage in combination with RA holds validity, as trxhere are reports that RXR agonists could have antidiabetic action in obese/diabetic rodents carries exciting promise [29]. These drugs working together with a PPAR-ligand could theoretically lower glucose more than either one alone. However, it is not clear from the studies done to date whether maximal costimulation of this heterodimeric receptor pair gives a larger glucose-lowering effect than does maximal stimulation of PPAR alone.

$\alpha$-Linolenic acid enhances adipogenic differentiation

The LA, the n-3 or omega- 3 polyunsaturated fatty acid is an essential poly unsaturated fatty acid, having potential medicinal applications. We reported earlier that LA stimulates adipocyte differentiation with ease in preadipocytes through acute induction in PPAR $\gamma, \mathrm{C} / \mathrm{EBP} \alpha$, and LPL mRNA expressions [13]. Accordingly, in the present experiment, enhanced expression of PPAR $\gamma$ was detected in all the three induction medium consisting of TZD. Although, the significant $(\mathrm{p}<0.01)$ upregulation in LA+TZD treatment showed than the other two treatments, which suggests the combination of LA along with TZD empowered the activation of PPAR $\gamma$ than the treatment with TZD alone. The expressions of adipocyte specific marker genes like PPAR $\gamma$, Adipo Q, LPL, and C/EBPa were analyzed and quantified by different methods to confirm the accountability of conversion of GMSC's.

PPAR $\gamma$, a member of PPARs that belongs to the nuclear hormone receptor superfamily, is abundantly expressed in all types of adipose tissue and its expression is sufficient enough for the initiation of adipogenesis [30]. TZD's produces adipogenic induction efficiently and could be appreciated for its function as a strong activator of PPAR $\gamma$ [31]. Besides, Adipo $\mathrm{Q}$, a secretory protein synthesized in the adipose tissue, plays a vital role in the lipids and carbohydrate metabolisms in muscle tissue by enhancing insulin sensitivity and fatty acid oxidation. Moreover, it is a vital element that reduces the expressions of gluconeogenic factors [32], thereby its declined expression in obesity or diabetic conditions is observed in humans, though it is predominantly expressed during the adipocyte differentiation [33]. However, our findings relate to the observation of the literature [33], where, higher expression of AdipoQ augmented the increased lipid accumulation in GMSC's (Figure 3A) during adipogenic differentiation.

$L P L$ gene was detected highly expressed throughout the adipocyte differentiation in our experiments, where LPL is an enzyme which regulates the fatty acid deposition and its metabolism in adipose tissue. The circulating triglycerides will be hydrolyzed by LPL and the fatty acids, which got released from this process will be taken up and stored in the adipose tissue [34]. In addition, LPL is an early marker for adipocyte differentiation, which is expressed independently of any inducers, suggesting that LPL expression blocks the cell proliferation and initiates adipocyte differentiation upon confluency [35]. Furthermore, the CCAAT/enhancer binding proteins (C/EBPs) are some of the first transcription factors found to be involved in the adipogenesis. The members of this family like $\mathrm{C} / \mathrm{EBPa}$, $\mathrm{C} / \mathrm{EBP} \beta$, and $\mathrm{C} / \mathrm{EBP} \delta$ along with PPAR $\gamma$ can induce the adipogenesis even in non-adipogenic cells [36] through a "feedforward" fashion. As in our results, upregulated expression of $\mathrm{C} / \mathrm{EBP} \alpha$ was observed in the final days of LA+TZD treatment, suggests terminal adipogenic differentiation. PPAR $\gamma$ and C/ EBPa will be highly expressed for the rest of the differentiation process and throughout the life of mature adipocytes [37]. Consequently, the joined expressions of all these genes, par- 
ticularly PPAR $\gamma$ and $\mathrm{C} / \mathrm{EBP} \alpha$ after the adipogenic inductions are sufficient enough to block the myogenic fate and to initiate the adipogenic differentiation in myosatellite cells [4]. Whereas the control myogenic samples, which was maintained without induction did not exhibit the adipogenic gene expressions, besides it retained the myogenic fate and formed myofibers, in vitro.

Moreover, the variations in the adipose specific gene expressions were visible according to the treatments. Where, the first treatment group was induced with TZD, which is a well known ligand for PPAR $\gamma$ receptor and found to be an effective inducer for adipocyte differentiation, in vitro $[4,38]$. Though in our study, its individual effect on adipogenic stimulation found to be less compared to the combined effect of TZD along with RA and LA. Interestingly, the combined TZD+RA treatment found upregulating the adipose specific genes, though the previous reports state RA as a potential adipogenic inhibitor [39]. Moreover, the study of the literature [38] used the similar combination, that is TZD+RA, and found RA suppressed the TZD mediated adipogenesis. In contrast to these findings, our result showed that the RA along with TZD does not inhibit but stimulates the expressions of adipose specific genes, and found to produce smaller adipocytes when compared to the LA in combination with TZD's.

\section{CONCLUSION}

Higher levels of free fatty acids cause enhanced insulin resistance in muscle and liver, however, its regulation through drugs like TZD's showed improved insulin sensitivity. Nevertheless, TZD's promotes PPAR $\gamma$ mediated adipocyte conversion in myoblasts. Our combined treatments of TZD's together with RA or LA showed significant fat cell transition of Korean black goat myosatellite cells though, the RA combination produced, comparatively, enormous amount of insulin sensitive smaller preadipocytes, whereas LA combination found to be producing fully differentiated large adipocytes, which are insulin resistant. Thus, we suggest that the RA in combination with TZD's may provide improved insulin sensitization in diabetic conditions as future approach in therapeutics.

\section{CONFLICT OF INTEREST}

We certify that there is no conflict of interest with any financial organization regarding the material discussed in the manuscript.

\section{ACKNOWLEDGMENTS}

S. Subi and S.J. Lee has contributed equally to this paper as first author. The research was fully supported and facilitated from National Research Foundation of Korea (120130344).

\section{REFERENCES}

1. Relaix F, Zammit PS. Satellite cells are essential for skeletal muscle regeneration: the cell on the edge returns centre stage. Development 2012;139:2845-56.

2. Lukjanenko L, Brachat S, Pierrel E, Lach-Trifilieff E, Feige JN. Genomic profiling reveals that transient adipogenic activation is a hallmark of mouse models of skeletal muscle regeneration. PLoS ONE 2013;8:e71084.

3. Yang YB, Pandurangan M, Jeong D, Hwang I. The effect of troglitazone on lipid accumulation and related gene expression in Hanwoo muscle satellite cell. J Physiol Biochem 2013;69: 97-109.

4. Singh NK, Chae HS, Hwang IH, et al. Transdifferentiation of porcine satellite cells to adipoblasts with ciglitizone. J Anim Sci 2007;85:1126-35.

5. Chambon P. A decade of molecular biology of retinoic acid receptors. FASEB J 1996;10:940-54.

6. Schug TT, Berry DC, Shaw NS, Travis SN, Noy N. Opposing effects of retinoic acid on cell growth result from alternate activation of two different nuclear receptors. Cell 2007;129: 723-33.

7. Danoviz ME, Reuveni ZY. Skeletal muscle satellite cells: background and methods for isolation and analysis in a primary culture system. Methods Mol Biol 2012;798:21-52.

8. Folgiero V, Migliano E, Tedesco M, et al. Purification and characterization of adipose-derived stem cells from patients with lipoaspirate transplant. Cell Transplant 2010;19:1225-35.

9. Vidal M, Kilroy G, Johnson J, et al. Cell growth characteristics and differentiation frequency of adherent equine bone marrowderived mesenchymal stromal cells: adipogenic and osteogenic capacity. Vet Surg 2006;35:601-10.

10. McNeil M. Adiopocyte staining with oil-red-O. Baton Rouge, LA, USA: Pennington Laboratory. Molecular Endocrinology, Pennington Biomedical Research Centre; 2005.

11. Ieronimakis N, Balasundaram G, Rainey S, et al. Absence of CD34 on murine skeletal muscle satellite cells marks a reversible state of activation during acute injury. PLoS ONE 2010; 5:e10920.

12. Suzawa K, Kobayashi M, Sakai Y, et al. Preferential induction of peripheral lymph node addressin on high endothelial venulelike vessels in the active phase of ulcerative colitis. Am J Gastroenterol 2007;102:1499-509.

13. Reza AM, Shiwani S, Singh NK, et al. Keratinocyte growth factor and thiazolidinediones and linolenic acid differentiate characterized mammary fat pad adipose stem cells isolated from prepubertal Korean black goat to epithelial and adipogenic lineage. In Vitro Cell Dev Biol Anim 2014;50:194-206.

14. Jankowski RJ, Deasy BM, Huard J. Muscle-derived stem cells. Gene Ther 2002;9:642-7.

15. Jesse TL, La Chance R, Iademarco MF, Dean DC. Interferon regulatory factor- 2 is a transcriptional activator in muscle where 
it regulates expression of vascular cell adhesion molecule-1. J Cell Biol 1998;140:1265-76.

16. Runembert I, Queffeulou G, Federici P, et al. Vimentin affects localization and activity of sodium-glucose cotransporter SGLT1 in membrane rafts. J Cell Sci 2002;115:713-24.

17. Gallanti A, Prelle A, Moggio M, et al. Desmin and vimentin as markers of regeneration in muscle diseases. Acta Neuropathol 1992;85:88-92.

18. Starkey JD, Yamamoto M, Yamamoto S, Goldhamer DJ. Skeletal muscle satellite cells are committed to myogenesis and do not spontaneously adopt nonmyogenic fates. J Histochem Cytochem 2011;59:33-46.

19. Ishibashi J, Perry RL, Asakura A, Rudnicki MA. MyoD induces myogenic differentiation through cooperation of its $\mathrm{NH} 2$ and COOH-terminal regions. J Cell Biol 2005;171:471-82.

20. Yablonka-Reuveni Z, Rivera AJ. Temporal expression of regulatory and structural muscle proteins during myogenesis of satellite cells on isolated adult rat fibers. Dev Biol 1994;164: 588-603.

21. Springer ML, Ozawa CR, Blau HM. Transient production of a-smooth muscle actin by skeletal myoblasts during differentiation in culture and following intramuscular implantation. Cell Motil Cytoskeleton 2002;51:177-86.

22. Talmadge RJ, Roy RR. Electrophoretic separation of rat skeletal muscle myosin heavy-chain isoforms. J Appl Physiol 1993;75: 2337-40.

23. Koch U, Lehal R, Radtke F. Stem cells living with a Notch. Development 2013;140:689-704.

24. Narkar VA, Downes M, Yu RT, et al. AMPK and PPARdelta agonists are exercise mimetics. Cell 2008;134:405-15.

25. Gervois PI, Torra P, Fruchart JC, Staels B. Regulation of lipid and lipoprotein metabolism by PPAR activators. Clin Chem Lab Med 2000;38:3-11.

26. Hallakou S, Doare L, Foufelle F, et al. Pioglitazone induces in vivo adipocyte differentiation in the obese Zuckerfa/fa rat. Diabetes 1997;46:1393-9.

27. Rebrin K, Steil GM, Mittelman SD, Bergman RN. Causal linkage between insulin suppression of lipolysis and suppression of liver glucose output in dogs. J Clin Invest 1996;
98:741-9.

28. Foellmi-Adams LA, Wyse BM, Herron D, Nedergaard J, Kletzien $\mathrm{RF}$. Induction of uncoupling protein in brown adipose tissue: synergy between norepinephrine and pioglitazone, an insulinsensitizing agent. Biochem Pharmacol 1996;52:693-701.

29. Mukherjee R, Davies PJ, Crombi DL, et al. Sensitization of diabetic and obese mice to insulin by retinoid $\mathrm{X}$ receptor agonists. Nature 1997;386:407-10.

30. Kubota N, Terauchi Y, Miki H, et al. PPAR $\gamma$ mediates highfat diet-induced adipocyte hypertrophy and insulin resistance. Mol Cell 1999;4:597-609.

31. Jarrar MH, Baranova A. PPAR $\gamma$ activation by thiazolidinediones (TZDs) may modulate breast carcinoma outcome: the importance of interplay with TGF $\beta$ signaling. J Cell Mol Med 2007; 11:71-87.

32. Dai LH, Xiong YZ, Deng CY, et al. Association of the A-G polymorphism in porcine adiponectin gene with fat deposition and carcass traits. Asian-Australas J Anim Sci 2006;19: 779-83.

33. Fu Y, Luo N, Klein RL, Garvey WT. Adiponectin promotes adipocyte differentiation, insulin sensitivity, and lipid accumulation. J Lipid Res 2005;46:1369-79.

34. Kolehmainen M, Vidal H, Ohisalo JJ, et al. Hormone sensitive lipase expression and adipose tissue metabolism show gender difference in obese subjects after weight loss. Int J Obes Relat Metab Disord 2002;26:6-16.

35. Gregoire FM, Smas CM, Sul HS. Understanding adipocyte differentiation. Physiol Rev 1998;78:783-809.

36. Darlington GJ, Ross SE, MacDougald OA. The role of C/EBP genes in adipocyte differentiation. J Biol Chem 1998;273:3005760 .

37. Rosen ED. The transcriptional basis of adipocyte development. Prostaglandins Leukot Essent Fatty Acids 2005;73:31-4.

38. Ohyama M, Matsuda K, Torii S, et al. The interaction between vitamin A and thiazolidinedione on bovine adipocyte differentiation in primary culture. J Anim Sci 1998;76:61-5.

39. Zizola CF, Frey SK, Jitngarmkusol S, et al. Cellular retinolbinding protein type I (CRBP-I) regulates adipogenesis. Mol Cell Biol 2010;30:3412-20. 